\title{
Endotracheale Intubation von Kindern
}

\author{
Alexander Humberg, Wolfgang Göpel
}

\begin{abstract}
Bei der Intubation von Kindern muss zügig gehandelt werden. Die anspruchsvolle Technik setzt viel Geschick voraus und kann selbst erfahrene Teams in schwierige Situationen bringen. Theoretisches Wissen über Besonderheiten im Kindesalter ist eine Grundvoraussetzung, um die Atemwege der jungen Patienten lege artis zu sichern.
\end{abstract}

Indikationen und Kontraindikationen | Die Intubation dient der Sicherung der Atemwege zur Ventilation und Oxygenierung des Patienten. Wenn möglich, sollten je nach klinischer Situation zunächst alle möglichen nichtinvasiven Verfahren zur Ventilation ausgeschöpft werden. Eine invasive Atemwegssicherung ist bei Kindern nur selten nötig. Untersuchungen zeigen, dass in der Notfallmedizin vor allem Kinder im Schulalter aufgrund eines Traumas intubiert werden müssen [3].

Kinder mit bekannt schwierigem Atemweg oder Dysmorphiesyndrom sollten möglichst mit anderen Maßnahmen oxygeniert werden.

Eine Intubation sollte dann nur mit Backup versucht werden - aber nur, wenn keine anderen Möglichkeiten der Ventilation bestehen und der Patient akut bedroht ist [5].

Erhöhte Versagerrate | Eine passende Gesichtsmaske und richtige Durchführung der Beatmung bietet in den meisten Situationen die lebensrettende Technik. Gelingt eine Intubation nicht, sollte spätestens nach dem zweiten misslungenen Intubationsversuch mittels Gesichts- oder Larynxmaske beatmet werden. Selbst erfahrene Notfallmediziner haben eine gegenüber der Intubation von Erwachsenen erhöhte Versagerrate.

Rachentubus oft ausreichend | Ziel einer Beatmung ist immer eine ausreichende Oxygenierung und Ventilation, so dass bei gut funktionierender Masken-Beutel-Beatmung diese nicht zwangsmäßig eskaliert werden muss. Die vulnerable Schleimhaut kann durch vermehrte Intubationsversuche anschwellen und zu Obstruktionen und konsekutiv unmöglicher Beatmung führen. Säuglinge können über die Anwendung eines Rachentubus oftmals ausreichend ventiliert werden. Nach etwas praktischer Übung lassen sich Larynxmasken einfach platzieren und erlauben eine oft ausreichende Beatmung des Kindes.

Larynxmaske empfohlen | Eine kürzlich erschienene gemeinsame Stellungnahme zum „Atem- wegsmanagement mit supraglottischen Atemwegshilfen in der Kindernotfallmedizin“ [4] empfiehlt die Larynxmaske neben dem Goldstandard der endotrachealen Intubation zur Oxygenierung und Ventilation von Kindern in Notfallsituationen. Bei Kindern unter $10 \mathrm{~kg}$ Körpergewicht konnte für die Verwendung eines Larynxtubus aufgrund schlechter Daten und möglicher Gefahren aktuell keine Empfehlung gegeben werden.

Risiken | Bei supraglottischen Atemwegshilfen ist das Riskio von Magenüberblähungen und damit auch für Regurgitation und Aspiration erhöht. Außerdem steigt das Risiko für eine mechanische Behinderung der Lunge mit Atelektasenbildung und reduzierter funktioneller Residualkapazität.

Aus diesem Grund sollte immer eine Larynxmaske mit Ösophaguskanal zur Anlage einer Magensonde gewählt werden.

Werden diese Punkte beachtet, ist die Beatmung mittels Larynxmaske ein sicheres Verfahren und eine gute Alternative zur endotrachealen Intubation.

Anamnese | Vor Intubation sollte man wichtige Punkte der Anamnese des Kindes erfragen, [1]:

- Geburtsanamnese und weitere Entwicklung des Kindes: Frühgeburtlichkeit? Bronchopulmonale Dysplasie?

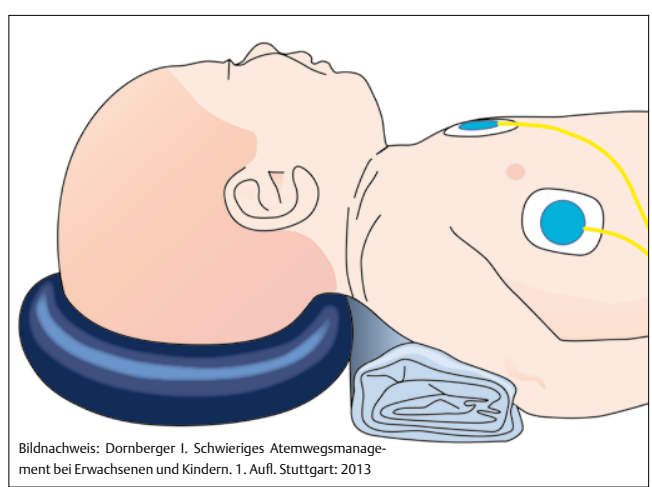

Abb. 1 Lagerung von Neugeborenen zur Intubation. 
Abb. 2 Lagerung und Reklination von größeren Kindern. Durch Reklination des Kopfes wird die Sicht auf die Stimmbandebene möglich $(\mathrm{O}=$ orale Achse, $\mathrm{P}=$ pharyngeale Achse, $\mathrm{T}=$ tracheale Achse).
- Anamnese respiratorischer Erkrankungen: Atemgeräusche? Schnarchen? Rhinitis? Infekte? Bronchiale Hyperreagibilität? Frequenz, Schweregrad und Charakter von Husten

- Wird in der Umgebung des Kindes geraucht?

- Bei kleineren Kindern: Fütterprobleme mit Aspirationen? Phonationsprobleme? Zeichen von Apnoen oder Zyanoseattacken?

- Vorangegangene Verletzungen oder Operationen im Bereich der Atemwege?

- Komplikationen bei vorangegangener Anästhesie?

- Neuromuskuläre Erkrankung oder kongenitale Anomalien bekannt?

Körperliche Untersuchung I Hier sollte vor allem auf folgende Stigmata geachtet werden:

- Auskultation und Untersuchung des Atemapparates

- vergrößerte Tonsillen

- Zeichen einer Laryngomalazie (inspiratorischer Stridor)

- lose Zähne (v.a. im Alter von 6-12 Jahren mit Milchgebiss)

- Dysmorphie (manibulare Hypoplasie, kleiner Mund, limitierte Mundöffnung, Syndrom, wie z.B. Pierre-Robin- oder Goldenhar-Syndrom)

$\begin{array}{ll}\text { Alter } & \text { Tubus } \\ \text { frühgeboren } & 2,0-3 \\ \text { 0-6 Monate } & 3-3,5 \\ \text { 6-12 Monate } & 3,5-4 \\ \text { 12-24 Monate } & 4-4,5 \\ \text { 2-4 Jahre } & 4,5-5,5 \\ \text { 4-6 Jahre } & 5-6 \\ \text { 6-10 Jahre } & 6-7 \\ \text { 10-16 Jahre } & 7-8\end{array}$

Tab. 1 Übersicht der geeigneten Größe des Tubus.

Aufklärung | Die Eltern und je nach Alter natürlich auch das Kind sollten über Ablauf und mögliche Komplikationen aufgeklärt werden.

\section{Anatomische und physiologische Besonderheiten im Kindesalter}

Neugeborene und Säuglinge | Bei Neugeborenen kann der große Hinterkopf und kurze Hals bei falscher Lagerung zu einer Obstruktion der oberen Atemwege führen. Die Kopflagerung kann optimiert werden, indem man den Kopf mit Hilfe einer Nackenrolle oder einem weichen Kopfring in Neutralposition bringt ( $\triangle$ Abb. 1).

Zu beachten ist ein physiologischer Larynxhochstand bei Säuglingen und Kleinkindern (Säugling auf Höhe von C2-C3, Kleinkinder C3-C4, Erwachsene (4-C5).

Ältere Kinder | Bei Kindern ab ca. 2 Jahren muss für eine bessere Darstellung der Glottis der Kopf rekliniert und leicht angehoben werden ( $\triangleright$ Abb. 2). Anders als beim adoleszenten Patienten befindet sich bis zum 8.-10. Lebensjahr die engste Stelle subglottisch [9]. Diese Engstelle kann auch nach problemloser Passage der Stimmritze während der Intubation ein weiteres Vorschieben des Tubus erschweren oder unmöglich machen.

Geringe Apnoetoleranz | Kinder sind gefährdet, unter länger dauerndem Intubationsmanöver oder insuffizienter Maskenbeatmung zügiger einen Sauerstoffsättigungsabfall zu erleiden (geringere Apnoetoleranz). Dies erklärt sich aus folgenden physiologischen Gegebenheiten [5]:

- erhöhter Sauerstoffbedarf (6-7 ml/ kg/min vs. 3-4 $\mathrm{ml} / \mathrm{kg} / \mathrm{min}$ beim Erwachsenen) durch den erhöhten Grundumsatz

- verminderte funktionelle Residualkapazität (FRC) im Verhältnis zur alveolären Ventilation und dadurch reduzierter Sauerstoffspeicher

- erhöhte „closing capacity“ und dadurch zügige Atelektasenbildung 
Nahrungskarenz | Für die elektive Intubation gilt die Nüchternheitsregel. Für Kinder bedeutet dies sechsstündige Nahrungskarenz für feste Nahrungen, vierstündige Karenz für Muttermilch und zweistündige für klare Flüssigkeiten (2-4-6-Stundenregel) [7].

\section{Benötigtes Material |}

- Sauerstoffanschluss mit Beatmungsbeutel und passender Gesichtsmaske (flach, weich und durchsichtig, eine Nummer größer und kleiner sollte zur Sicherheit vorgehalten werden)

- getestete Absaugeinheit mit Katheter

- Laryngoskop und passende Spatel, Ersatzbatterien

- Tuben in passender Größe und in einer Nummer größer und kleiner

- Führungsstab für den Tubus

- Stethoskop

- EKG- und Pulsoxymetrie - Monitoring mit sauerstoffsättigungsabhängigen Herzfrequenzton

- Kapnografie oder-metrie

- bei geblockten Tuben: Cuffdruckmessung

- Material zur Fixierung des Tubus

- individuelle Einstellung des Beatmungsgeräts für das jeweilige Kind

- Medikamente zur Narkoseeinleitung und Notfallmedikamente in der richtigen Dosierung aufgezogen, sowie mindestens ein sicherer venöser Zugang

- mind. eine erfahrene assistierende Person

- bei Dysmorphiesyndromen oder bei erwartetem schwierigen Atemweg Vorhalten einer qualifizierten Assistenz (pädiatrische Intensivmedizin, Anästhesie, HNO, Kinderchirurgie u.a.)

Cave Für den Notfall muss geklärt sein, wer als Backup hinzugerufen werden kann.

Wahl der richtigen Tubusgröße I Die Wahl der richtigen Tubusgröße ist entscheidend für Komplikationen vor und nach Extubation.

Ist der Tubus zu groß, kann er erhebliche Schäden mit Langzeitfolgen anrichten. Ist er zu klein, besteht ein unnötig erhöhter Atemwegswiderstand.

$\mathrm{Zu}$ beachten ist allerdings, dass bei klinischen Hinweisen auf einen engeren oder größeren Atemweg (Widerstand beim intubieren, hoher Anteil an Nebenluft beim beatmen) ein anderer Tubus gewählt wird. Die Abschätzung der Tubusgröße jenseits des ersten Lebensjahres kann mit Hilfe der Formel ID $(\mathrm{mm})=4,5+($ Alter $($ Jahre $) / 4$ ) vereinfacht werden (Formel für heutzutage dünnwandigere Tuben modifiziert). Für geblockte Tuben kann eine halbe Größe kleiner gewählt werden. > Tab. 1 gibt eine Übersicht der Größen je nach Alter.

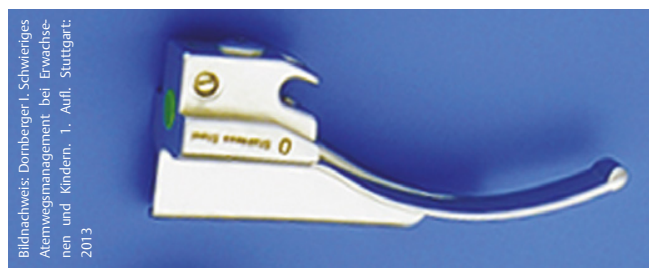

Abb. 3 Macintosh-Spatel
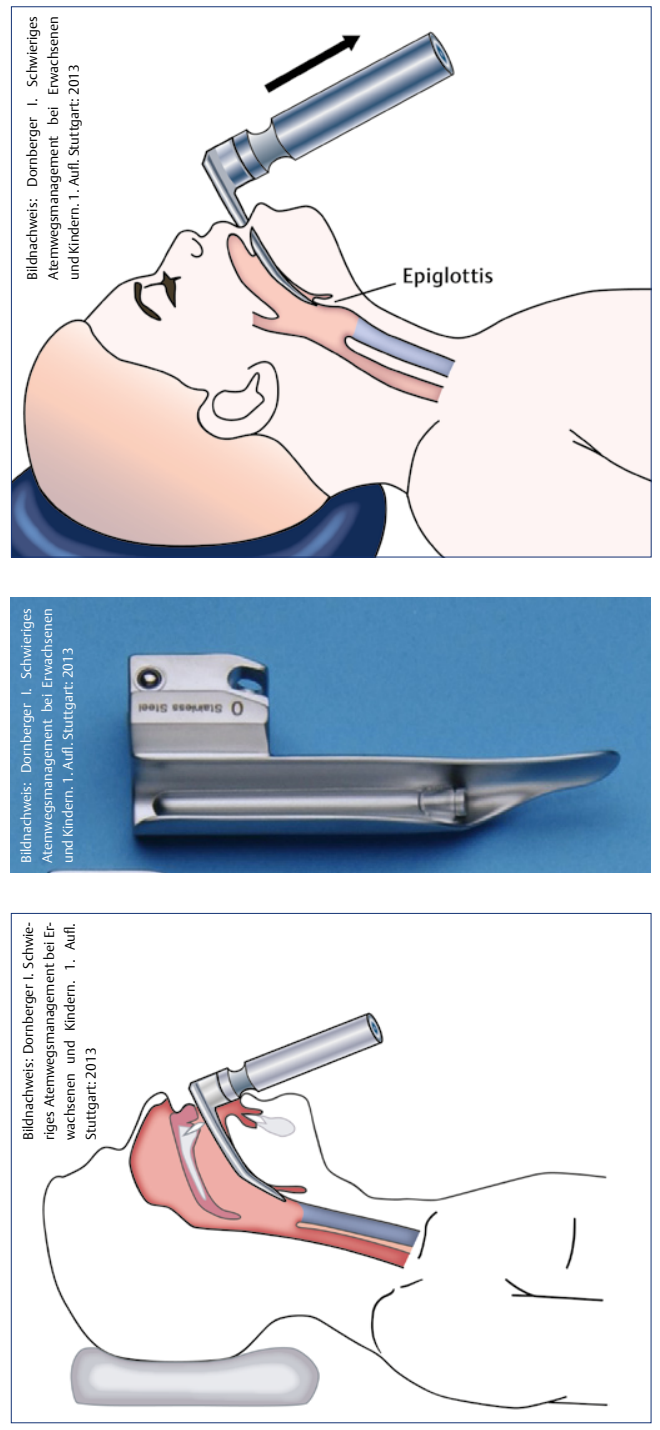

Abb. 4 Aufstellen der Epiglottis mit dem Macintosh-Spatel.

Abb. 5 Miller-Spatel

Abb. 6 Aufladen der Epiglottis mit dem MillerSpatel.
Geblockter oder ungeblockter Tubus | Aufgrund der subglottischen Stenose sind Drucknekrosen der Schleimhaut in diesem Bereich durch die Benutzung gecuffter Tuben gefürchtet. Tuben mit Cuff haben allerdings den Vorteil, vermehrte Intubationsversuche bis zum Finden des passenden Tubus zu reduzieren oder Probleme mit Luftleckage und das Risiko von Aspirationen zu vermindern. Bei kurzer Verweildauer ist es heutzutage möglich, gecuffte Tuben unter strenger Beachtung der Cuffdrücke jenseits der Neugeborenenperiode zu benutzen [5]. Der Cuffdruck sollte $20 \mathrm{cmH}_{2} \mathrm{O}$ nicht überschreiten.

Wahl der Tubustiefe I Diese sollte an klinischen Parametern und nicht an starren Tiefenvorgaben gewählt werden. Eine seitengleiche Auskultation 
Abb. 7 BURP-Manöver beim Säugling.

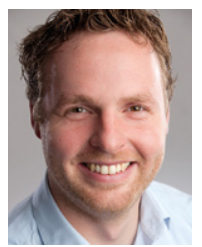

Dr. med. Alexander Humberg

ist Assistenzarzt in der Neonatologie und Pädiatrischen Intensivmedizin an der Klinik für Kinder- und Jugendmedizin am Universitätsklinikum Schleswig-Holstein, Campus Lübeck. alexander.humberg@uksh. de

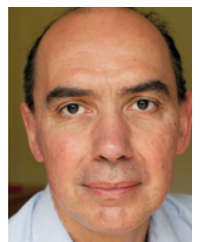

Prof. Dr. med. Wolfgang Göpel

ist Ärztlicher Leiter der Neonatologie und Pädiatrischen Intensivmedizin an der Klinik für Kinder- und Jugendmedizin am Universitätsklinikum Schleswig-Holstein, Campus Lübeck. wolfgang.goepel@uksh.de

Interessenkonflikt

Die Autoren geben an, dass kein Interessenkonflikt besteht.

DOI 10.1055/s-0042-113304 Dtsch Med Wochenschr 2016; 141: 1409-1412 (c) Georg Thieme Verlag KG . Stuttgart · New York . ISSN 0012-0472

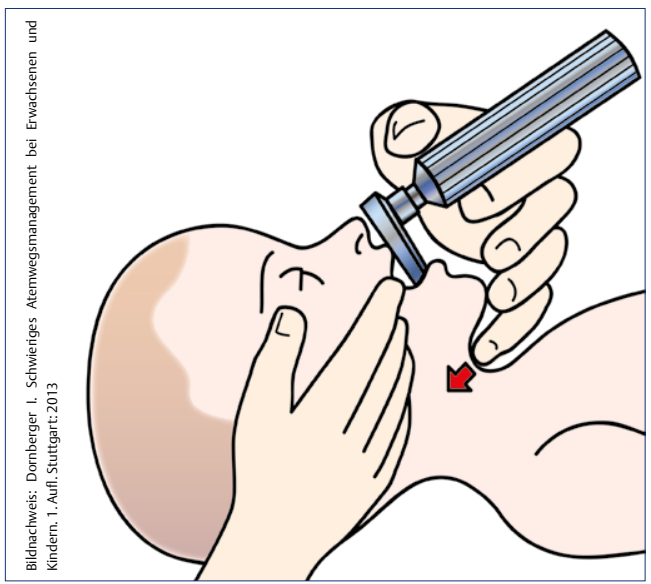

sowie der radiologische Nachweis der Tubusspitze oberhalb der Carina (bzw. auf Höhe des zweiten Brustwirbels) dienen dem Nachweis einer korrekten Lage.

Macintosh-Spatel | Dieser Spatel hat einen leicht gebogenen Verlauf ( Abb. 3). Zur Intubation wird der Spatel hinter die Epiglottis in die Vallecula epiglottica geführt und diese dadurch aufgestellt $(\triangleright$ Abb. 4$)$.

Miller-Spatel | Unter Narkose kann der weiche Gaumen und die weiche U-förmige Epiglottis den Atemweg verlegen und die Intubation erschweren. Hier kann ein gerader Laryngoskopspatel hilfreich sein. Der Miller-Spatel ( $\triangle$ Abb. 5 ) hat einen geraden Verlauf und vereinfacht die Aufladung der Epiglottis bei kleineren Kindern ( $\bullet$ Abb. 6 ).

\section{Praktische Durchführung und Ablauf}

Vorschieben des Spatels | Zur Intubation wird das Kind wie oben genannt gelagert und nach ausreichender Präoxygenierung und Gabe der Narkotika der Spatel in den Mundraum eingeführt. Bei der Präoxygenierung von Kindern ist im Gegensatz zur Präoxygenierung von Erwachsenen eine deutlich geringere Hypoxietoleranz zu beachten, die oftmals „Zwischenbeatmungen“ bei der Narkoseeinleitung erfordert. Für die Intubation wird die Zunge nach links geschoben und der Spatel mittig gehalten. Unter Sicht wird der Spatel vorsichtig in Richtung Glottis geschoben und je nach Spatel die Epiglottis aufgeladen oder aufgestellt. Um den Raum zu vergrößern, hebt der Intubateur den Unterkiefer mit Hilfe des Laryngoskops hoch (nicht hebeln!) in Richtung Decke.

BURP-Manöver | Mithilfe von Druck von außen auf den Kehlkopf (BURP-Manöver = backward upward rightward pressure), kann in manchen Fällen die Sicht auf die Glottis verbessert werden. Bei kleinen Kindern kann das BURP-Manöver mittels des kleinen Fingers der Hand am Laryngoskop durchgeführt werden ( $\triangleright$ Abb. 7).
Vorschieben des Tubus | Nun kann der Tubus mittig durch die Stimmritze geschoben werden. An der ventralen Wand der Trachea ragt der Ringknorpel wulstig in das Lumen. Manchmal kann hier durch Drehen des Tubus das Vorschieben vereinfacht werden. Die schwarze Markierung sollte hinter der Glottis zu liegen kommen, blockbare Tuben werden nun geblockt. Die Tubusmarkierung an der Zahnreihe wird registriert und dokumentiert. Nun erfolgt die Lagekontrolle des Tubus (Auskultation [symmetrisches Atemgeräusch?] Pulsoxymetrie, bds. Heben und Senken des Thorax unter Beatmung, Kapnometrie und ggfs. -grafie [Goldstandard], ggfs. Röntgen Thorax).

\section{Komplikationen und Nachsorge}

Komplikationen | Mögliche Komplikationen sind:

- Bradykardie und Herzstillstand durch Vagusreiz

- Fehlintubation des Ösophagus

- Aspiration

- Verletzung der Stimmbänder

- akzidentelle Einlungenbeatmung (bei zu tiefer Intubation)

- Nekrosen und Ulzerationen der Schleimhaut bei zu hohem Druck auf das Gewebe (zu großer Tubus oder zu hoher Cuffdruck)

- Zahnschäden

- Laryngospasmus

Die Strukturen des kindlichen Atemwegs sind weicher als beim Erwachsenen, weshalb narkotisierte Kinder anfälliger für einen Atemwegskollaps sind [1].

Subglottische Stenosen I Kinder sind aufgrund der Größe und der Anlage des Krikoids bei rezidivierenden Intubationen gefährdet, subglottische Stenosen zu entwickeln. Gerade mehrfache oder traumatische Intubationen, hoher Cuffdruck und lange Intubationsdauer sind Risikofaktoren [6].

Cave Minimale Bewegungen des Kopfes können zu unbeabsichtigten Extubationen oder Verlagerungen des Tubus in tiefere Regionen der Atemwege führen [8] und die Beatmung erschweren.

Konsequenz für Klinik und Praxis

- Säuglinge und Kleinkinder haben einen ein physiologischen Larynxhochstand.

- Bei Kindern bis zum 8.-10. Lebensjahr befindet sich die engste Stelle subglottisch.

- Ist der Tubus zu groß, kann er erhebliche Schäden mit Langzeitfolgen anrichten. Ist er zu klein, besteht ein unnötig erhöhter Atemwegswiderstand.

- Narkotisierte Kinder sind anfälliger für einen Atemwegskollaps.

Vollständiges Literaturverzeichnis unter http://dx.doi.org/10.1055/s-0042-113304 


\section{Literatur}

1 Adewale L. Anatomy and assessment of the pediatric airway. Paediatr Anaesth 2009; 19: 1-8

2 Dornberger I. Schwieriges Atemwegsmanagement bei Erwachsenen und Kindern. Stuttgart: Georg Thieme Verlag 2013

3 Eich C, Roessler M, Nemeth et al. Characteristics and outcome of prehospital paediatric tracheal intubation attended by anaesthesia-trained emergency physicians. Resuscitation 2009; 80: 1371-77

4 Keil J, Jung P, Schiele A, Urban B et al. Interdisciplinary consensus statement on alternative airway management with supraglottic airway devices in pediatric emergency medicine. Anaesthesist 2015; 65: 57-66

5 Russo SG, Russo SG, Trieschmann U et al. Airway management in paediatric emergencies. Notfall Rettungsmed 2014; 17: 105-112
6 Schultz-Coulon $\mathrm{HJ}$. The management of postintubation stenoses in children. HNO 2004; 52 . 363-378

7 Smith I, Kranke P, Murat I et al. Perioperative fasting in adults and children: guidelines from the European Society of Anaesthesiology. Eur J Anaesthesiol 2011; 28: 556-569

8 Weiss M, Knirsch W, Kretschmar O et al. Tracheal tube-tip displacement in children during head-neck movement--a radiological assessment. $\mathrm{Br}$ ] Anaesth 2006. 96: 486-91

9 Höhne C, Haack M, Machotta A, Kaisers U. Airway management in pediatric anesthesia. Anaesthesist 2006; 809-819 